\title{
Phase Equilibria at Ti-Al Interface Under Low Oxygen Pressure
}

\author{
Chien Chon Chen
}

Department of Energy Engineering, National United University, Miaoli 36003, Taiwan

Received: October 17, 2013 / Accepted: December 20, 2013

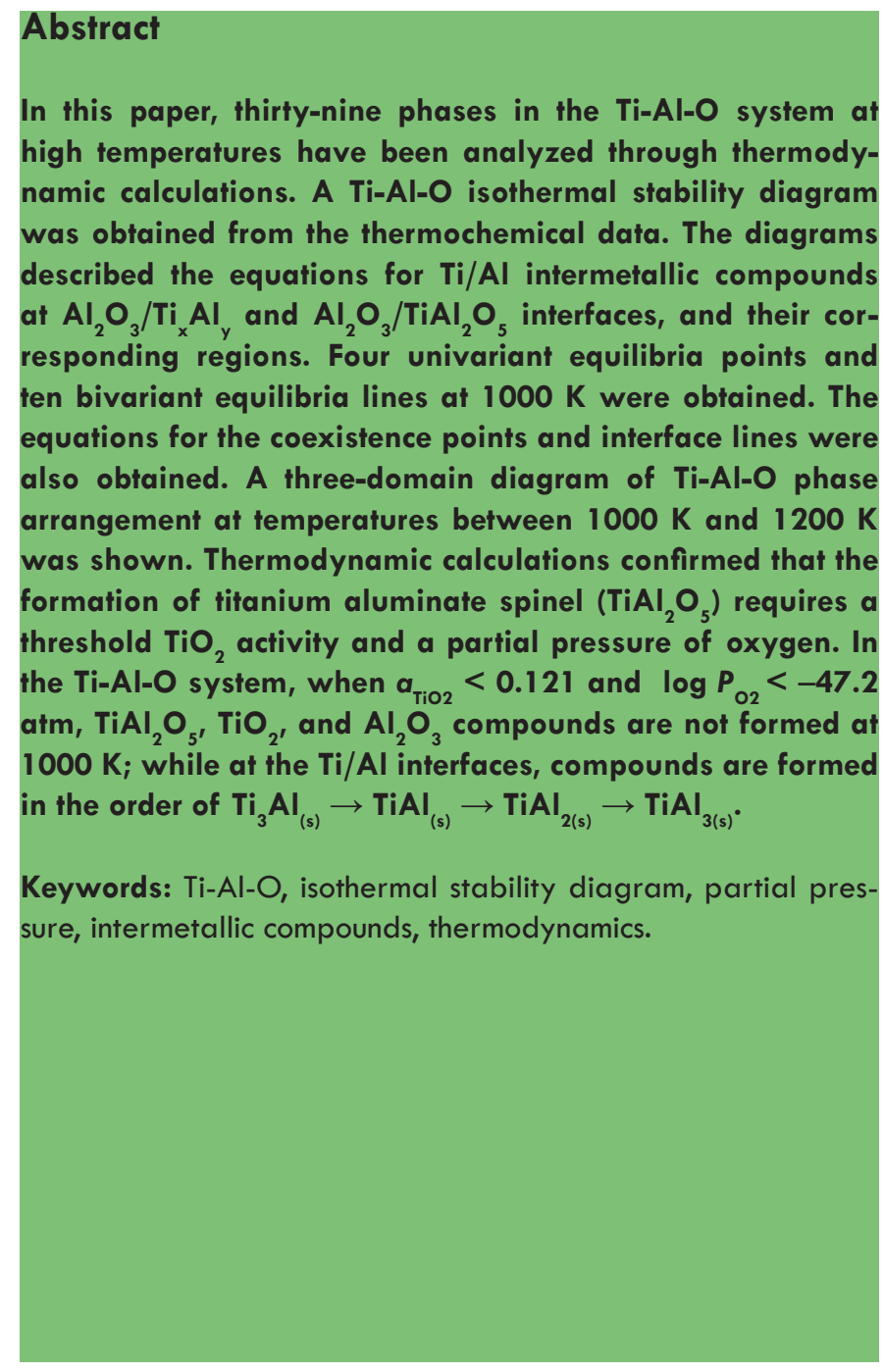

*Corresponding author: chentexas@gmail.com

\section{Introduction}

Titanium-aluminide compounds, known as ordered intermetallic compounds, are potential candidates for elevated temperature applications in the aerospace and automotive industries because of their light weight, high specific strength, high specific modulus, and good creep resistance (Chen et al., 2009; Zhong et al., 2012; Wang et al., 2013).The intermetallic compounds of $\mathrm{Ti}$-Al alloys include $\mathrm{Ti}_{3} \mathrm{Al}, \mathrm{TiAl}, \mathrm{TiAl}_{2}$, and $\mathrm{TiAl}_{3}$ (Charlu et al., 1974; Chen et al., 1995; Braun et al., 2001). They possess attractive properties of relatively low density, high melting point, high thermal conductivity, high specific strength, and resistance to oxidation at elevated temperatures. The Ti-Al alloys, with a duplex $\left(\mathrm{Ti}_{3} \mathrm{Al}+\mathrm{TiAl}\right)$ lamellar microstructure, exhibited improved room-temperature ductility while maintaining adequate hightemperature creep rates and oxidation resistance (Ward-Close et al., 1995; Lin et al., 2000; Cheng et al., 2013).

$\mathrm{Ti} / \mathrm{Al}_{2} \mathrm{O}_{3}$ interface reactions have been extensively investigated in the literature (Lu et al., 1995a; Lu et al., 1995b; Dehm et al., 1998; Zalar et al., 1999; Feng et al., 2012). However, some controversies remained about the mechanism and formation sequence of the interface reaction products, since different experimental constructions may affect both thermodynamic and kinetic conditions and thus the diffusion path in the Ti-Al-O system. For example, Li (Li et al., 1992) suggested two possible diffusion paths at $1100{ }^{\circ} \mathrm{C}: \mathrm{Al}_{2} \mathrm{O}_{3} / \mathrm{TiAl} / \mathrm{Ti}_{3} \mathrm{Al} / \alpha-\mathrm{Ti} / \beta-\mathrm{Ti}$, for an infinite Ti supply from thick Ti foils; or $\mathrm{Al}_{2} \mathrm{O}_{3} / \mathrm{Ti}_{3} \mathrm{Al} / \alpha-\mathrm{Ti} / \beta-\mathrm{Ti}$, for a finite Ti supply from thin Ti foils. Additionally, the oxides of $\mathrm{TiO}_{2}$ and $\mathrm{TiAl}_{2} \mathrm{O}_{5}$ could be formed in the Ti-Al system. Their formation depends on the partial pressure of oxygen and the activity of titanium. The mechanisms of formation of the titanium aluminate spinel $\left(\mathrm{TiAl}_{2} \mathrm{O}_{5}\right)$ in the $\mathrm{TiO}_{2}-\mathrm{Al}_{2} \mathrm{O}_{3}$ interface under 1 bar oxygen partial pressure have been investigated in Seifert's studies (Seifert et al., 2001). Xu et al. (2006) found that $\mathrm{TiAl}_{3^{\prime}}$ $\mathrm{Ti}_{2} \mathrm{Al}_{5}, \mathrm{TiAl}_{2}, \mathrm{TiAl}$, and $\mathrm{Ti}_{3} \mathrm{Al}$ could be formed by a Ti/Al diffusion 
couple experimental process. Cai et al. (2006) fabricated Al/ $\mathrm{TiO}_{2}, \mathrm{Ti}(\mathrm{Al}, \mathrm{O}) / \mathrm{Al}_{2} \mathrm{O}_{3}, \mathrm{Ti}_{3} \mathrm{Al}(\mathrm{O}) / \mathrm{Al}_{2} \mathrm{O}_{3}$ and $\mathrm{TiAl} / \mathrm{Al}_{2} \mathrm{O}_{3}$ composites by high energy mechanical milling using $\mathrm{Al}, \mathrm{TiO}_{2}, \mathrm{Ti}$, and $\mathrm{Al}_{2} \mathrm{O}_{3}$ powders as the starting materials.

According to the literature (Kainuma et al., 1994; Gerasimov et al., 1996; Kostov et al., 2008; Pang et al., 2013), several parameters influence $\mathrm{Ti} / \mathrm{Al}$ interface conditions, such as temperature, partial pressure of oxygen, activity of Ti, activity of compounds, Gibbs free energy, activation energy, heat treatment time, and diffusion coefficient. A convenient method of representing the thermodynamic information involves the construction of isothermal diagrams showing the range of gas compositions, over which a condensed phase can exist either by itself or in equilibrium with another condensed phase. This type of diagram is called an isothermal stability diagram. It can be more useful to apply an isothermal stability diagram in discussing gas-condensed phases at a constant temperature, rather than a general phase diagram of temperature-composition. This work considers the effects of temperature, partial oxygen pressure, activities of $\mathrm{Ti}$ and $\mathrm{TiO}_{2}$, and Gibbs free energy for thermodynamic calculations the Ti-Al-O system. The mechanisms that operate in the Ti$\mathrm{Al}-\mathrm{O}$ system at high temperatures are thereby clarified. The purpose of the present work is to explain and locate the compounds in Ti-Al-O system by an isothermal stability diagram, which provides guidelines to obtain titanium-aluminide compounds.

\section{Thermodynamic Calculations and Results}

\section{Phases in the Ti-Al-O System}

In order to simplify the calculation and the final diagram we ignore the dominant phase in the Ti-O system (TixOy) which has a non-stoichiometry. The phase diagrams (Figure 1) of Al-O (Wriedt, 1985; Copland, 2006), Ti-O, Ti-Al, and $\mathrm{TiO}_{2}-\mathrm{Al}_{2} \mathrm{O}_{3}$ (Caron et al., 1996), and thermodynamic data books (Pourbaix, 1974; Chase et al., 1985; Barin, 1989) show 31 different condensed phases and 8 gaseous phases in the Ti-Al-O system. The 39 phases are as follows: $\mathrm{Al}(\mathrm{I}), \mathrm{Al}_{(\mathrm{g})^{\prime}} \mathrm{Ti}_{(\alpha)^{\prime}} \mathrm{Ti}_{(\beta)^{\prime}} \mathrm{Ti}_{(\mathrm{g})^{\prime}}$ $\left.\mathrm{O}_{2(\mathrm{~g})^{\prime}} \mathrm{Al}_{2} \mathrm{O}_{3(\alpha,}\right), \mathrm{Al}_{2} \mathrm{O}_{3(\mathrm{k})^{\prime}} \mathrm{Al}_{2} \mathrm{O}_{3(\delta)^{\prime}}, \mathrm{Al}_{2} \mathrm{O}_{3(\gamma)^{\prime}}, \mathrm{Al}_{2} \mathrm{O}_{2(\mathrm{~g})^{\prime}} \mathrm{Al}_{2} \mathrm{O}_{(\mathrm{g})^{\prime}}, \mathrm{AlO}_{2(\mathrm{~g})^{\prime}}$ $\mathrm{AlO}_{(\mathrm{g})^{\prime}} \mathrm{TiO}_{(\mathrm{g})^{\prime}}, \mathrm{TiO}_{(\alpha)^{\prime}} \mathrm{TiO}_{(\beta)^{\prime}}, \mathrm{TiO}_{(\gamma)^{\prime}} \mathrm{TiO}_{2(s)^{\prime}} \mathrm{Ti}_{2} \mathrm{O}_{(\mathrm{s})^{\prime}} \mathrm{Ti}_{2} \mathrm{O}_{3(\alpha)^{\prime}} \mathrm{Ti}_{2} \mathrm{O}_{3(\beta)^{\prime}}$ $\mathrm{Ti}_{3} \mathrm{O}_{(\mathrm{s})^{\prime}} \mathrm{Ti}_{3} \mathrm{O}_{2(\mathrm{~s})^{\prime}}, \mathrm{Ti}_{3} \mathrm{O}_{5(\mathrm{~s})^{\prime}} \mathrm{Ti}_{3} \mathrm{O}_{5(\beta)^{\prime}} \mathrm{Ti}_{4} \mathrm{O}_{7(\mathrm{~s}))^{\prime}} \mathrm{Ti}_{5} \mathrm{O}_{9(\mathrm{~s})^{\prime}} \mathrm{Ti}_{6} \mathrm{O}_{11(\mathrm{~s})^{\prime}} \mathrm{Ti}_{8} \mathrm{O}_{15(\mathrm{~s})^{\prime}}$ $\mathrm{Ti}_{9} \mathrm{O}_{17(s)^{\prime}} \mathrm{Ti}_{16} \mathrm{O}_{31(s)^{\prime}} \mathrm{Ti}_{50} \mathrm{O}_{99(s)^{\prime}}, \mathrm{TiAl}_{(\mathrm{s})^{\prime}}, \mathrm{TiAl}_{2(\mathrm{~s})^{\prime}} \mathrm{TiAl}_{3(\mathrm{~s})^{\prime}} \mathrm{Ti}_{2} \mathrm{Al}_{5(\mathrm{~s})^{\prime}}, \mathrm{Ti}_{3} \mathrm{Al}_{(\mathrm{s})^{\prime}}$ and $\mathrm{TiAl}_{2} \mathrm{O}_{5(\mathrm{~s})}$. Here, the subscripts $\mathrm{g}$, s and represents gas and solid, respectively, and other scubscripts, different phases. To simplify the calculation of phases in Ti-Al-O system, the 7 gaseous species $\mathrm{Al}_{(\mathrm{g})^{\prime}} \mathrm{Ti}_{(\mathrm{g})^{\prime}} \mathrm{Al}_{2} \mathrm{O}_{2(\mathrm{~g})^{\prime}} \mathrm{Al}_{2} \mathrm{O}_{(\mathrm{g})^{\prime}} \mathrm{AlO}_{2(\mathrm{~g})^{\prime}} \mathrm{AlO}_{(\mathrm{g})^{\prime}}$ and $\mathrm{TiO}_{(\mathrm{g})}$ with the exception of oxygen, will be ignored at first because of their low partial vapor pressure within the $\mathrm{Al}-\mathrm{O}$ and $\mathrm{Ti}-\mathrm{O}$ systems. Moreover, because the condensed phase $\mathrm{Al}_{2} \mathrm{O}_{3}(\alpha)(\Delta \mathrm{G}=$ $-1361.42 \mathrm{~kJ} / \mathrm{mol}$ at $1000 \mathrm{~K}$ ) (Chase et al., 1985) is much more stable, the other 3 alumina phases of $\mathrm{Al}_{2} \mathrm{O}_{3(\alpha)^{\prime}} \mathrm{Al}_{2} \mathrm{O}_{3(\kappa)^{\prime}}, \mathrm{Al}_{2} \mathrm{O}_{3(\delta)}$ will also be ignored. In addition, the 13 condensed phases of $\mathrm{TiO}_{(\beta)^{\prime}} \mathrm{TiO}_{(\gamma)}, \mathrm{Ti}_{2} \mathrm{O}_{(s)^{\prime}}, \mathrm{Ti}_{2} \mathrm{O}_{3(\beta)^{\prime}} \mathrm{Ti}_{3} \mathrm{O}_{(s)^{\prime}}, \mathrm{Ti}_{3} \mathrm{O}_{2(s)^{\prime}} \mathrm{Ti}_{3} \mathrm{O}_{5(\beta)^{\prime}}, \mathrm{Ti}_{5} \mathrm{O}_{9(s)^{\prime}}$ $\mathrm{Ti}_{6} \mathrm{O}_{11(\mathrm{~s})^{\prime}} \mathrm{Ti}_{8} \mathrm{O}_{15(\mathrm{~s})^{\prime}} \mathrm{Ti}_{16} \mathrm{O}_{31(\mathrm{~s})^{\prime}} \mathrm{Ti}_{9} \mathrm{O}_{17(\mathrm{~s})^{\prime}}$ and $\mathrm{Ti}_{50} \mathrm{O}_{99(\mathrm{~s})}$ will also be ignored because they are less stable than $\mathrm{TiO}_{(\alpha)^{\prime}} \mathrm{TiO}_{2(s)^{\prime}} \mathrm{Ti}_{2} \mathrm{O}_{3(\alpha)^{\prime}}$ $\mathrm{Ti}_{3} \mathrm{O}_{5(\alpha)^{\prime}}$ and $\mathrm{Ti}_{4} \mathrm{O}_{7(\mathrm{~s})}$ in the Ti-O system. As a result, the Ti-Al-O isothermal stability diagram now only includes 15 condensed phases, i.e., $\mathrm{Al}_{(s, 1)^{\prime}} \mathrm{Ti}_{(\alpha)^{\prime}} \mathrm{Ti}_{(\beta)}, \mathrm{Al}_{2} \mathrm{O}_{3(\alpha)^{\prime}} \mathrm{TiO}_{(\alpha)^{\prime}} \mathrm{TiO}_{2(\mathrm{~s})^{\prime}} \mathrm{Ti}_{2} \mathrm{O}_{3(\alpha)^{\prime}} \mathrm{Ti}_{3} \mathrm{O}_{5(\alpha)^{\prime}}$ $\mathrm{Ti}_{4} \mathrm{O}_{7(s)^{\prime}}, \mathrm{TiAl}_{(\mathrm{s})^{\prime}}, \mathrm{TiAl}_{2(\mathrm{~s})^{\prime}}, \mathrm{TiAl}_{3(\mathrm{~s})^{\prime}}, \mathrm{Ti}_{2} \mathrm{Al}_{5(\mathrm{~s}))^{\prime}}, \mathrm{Ti}_{3} \mathrm{Al}_{(\mathrm{s})^{\prime}}, \mathrm{TiAl}_{2} \mathrm{O}_{5(\mathrm{~s})^{\prime}}$ and one gas phase of oxygen which will be investigated by thermochemical data and thermodynamic calculations. The 16 phases used for Ti-Al-O isothermal stability diagram construction in the temperature range of 900 to $1200 \mathrm{~K}$ are shown in Table 1.

\section{Gaseous Species}

As discussed above, gas species other than oxygen were ignored in developing the isothermal stability diagram. However, vapor pressures in the solid and liquid phases are important in $\mathrm{Al}-\mathrm{O}$ and Ti-O systems at high temperature. We have discussed $\mathrm{Ni}-\mathrm{Al}-\mathrm{O}$ system in a previous paper (Kuo et al., 2009). In this section, we will discuss the reactions in the gas phase in the Ti-O system. The equilibrium partial vapor pressure of titanium and oxygen in $\mathrm{TiO}_{(\mathrm{s})}$ can be calculated from the Gibbs free energy as follows:

$\mathrm{TiO}_{(\mathrm{s})} \rightarrow \mathrm{Ti}_{(\mathrm{g})}+\frac{1}{2} \mathrm{O}_{2(\mathrm{~g})}, \quad \Delta G_{1}^{o}=4.97-\frac{28262.2}{T}(\mathrm{~kJ} / \mathrm{mol}) \quad[26]$

The standard molar free energy change in this reaction is given by $\Delta G^{0}{ }_{1}=-R T \times \ln K_{1}$. Here $K_{1}$ can be expressed as:

$$
K_{1}=\frac{P_{\mathrm{Ti}} \cdot\left(P_{\mathrm{O}_{2}}\right)^{0.5}}{a_{\mathrm{TiO}}}
$$

where $a_{\mathrm{TiO}}, P_{\mathrm{Ti}}$ and $P_{\mathrm{O} 2}$ represent the activity of TiO, partial pressure of $\mathrm{Ti}$, and partial pressure of oxygen, respectively. Since the activity of a solid is a unity, the relationship between $P_{\mathrm{Ti}}$ and $P_{\mathrm{O} 2}$ can be represented as:

$$
\log P_{\mathrm{O}_{2(\mathrm{~g})}}=2\left(\log K-\log P_{\mathrm{Ti}}\right)
$$

Additionally,

$$
\log K=\frac{-\Delta G_{1}^{o}}{2.303 R T}
$$

In Equ. $4, K$ and $R$ are two constants; therefore, $\Delta G^{0}$ is only a function of temperature $T$. The $\Delta G^{0}$ can be express as $\Delta G^{0}=A$ $+B T$, where $A$ and $B$ are two numerical numbers. Based on the preceding discussion and thermodynamic data books (Pourbaix 1974; Chase et al., 1985; Barin 1989), the vapor pressures of $\mathrm{Ti}_{(\mathrm{g})}$ in $\mathrm{Ti}_{(\mathrm{s})^{\prime}} \mathrm{TiO}_{(\mathrm{g})}$ in $\mathrm{TiO}_{(\mathrm{s})^{\prime}}, \mathrm{TiO}_{(\mathrm{g})}$ in $\mathrm{Ti}_{3} \mathrm{O}_{5(\mathrm{~s})^{\prime}} \mathrm{TiO}_{(\mathrm{g})}$ in $\mathrm{Ti}_{4} \mathrm{O}_{7(\mathrm{~s})^{\prime}}$ and $\mathrm{TiO}_{(\mathrm{g})}$ in $\mathrm{TiO}_{2(\mathrm{~s})}$ are:

$$
\begin{aligned}
& \left.\mathrm{Ti}_{(\mathrm{s})} \rightarrow \mathrm{Ti}_{(\mathrm{g})} \quad \Delta G_{2}^{o}=464.9-0.138 \mathrm{(kJ} / \mathrm{mol}\right) \\
& \mathrm{TiO}_{(\mathrm{s})} \rightarrow \mathrm{TiO}_{(\mathrm{g})} \quad \Delta G_{3}^{o}=43.8-0.085 T(\mathrm{~kJ} / \mathrm{mol}) \\
& \left.\mathrm{Ti}_{3} \mathrm{O}_{5(\mathrm{~s})} \rightarrow 3 \mathrm{TiO}_{(\mathrm{g})}+\mathrm{O}_{2(\mathrm{~g})} \Delta G_{4}^{o}=2581.2-0.70 T \mathrm{~kJ} / \mathrm{mol}\right) \\
& \mathrm{Ti}_{4} \mathrm{O}_{7(\mathrm{~s})} \rightarrow 4 \mathrm{TiO}_{(\mathrm{g})}+\frac{3}{2} \mathrm{O}_{2(\mathrm{~g})} \Delta G_{5}^{o}=3568.1-0.961(\mathrm{~kJ} / \mathrm{mol}) \\
& \mathrm{TiO}_{2(\mathrm{~s})} \rightarrow \mathrm{TiO}_{(\mathrm{g})}+\frac{1}{2} \mathrm{O}_{2(\mathrm{~g})} \quad \Delta G_{6}^{o}=984.2-0.266(\mathrm{~kJ} / \mathrm{mol})
\end{aligned}
$$




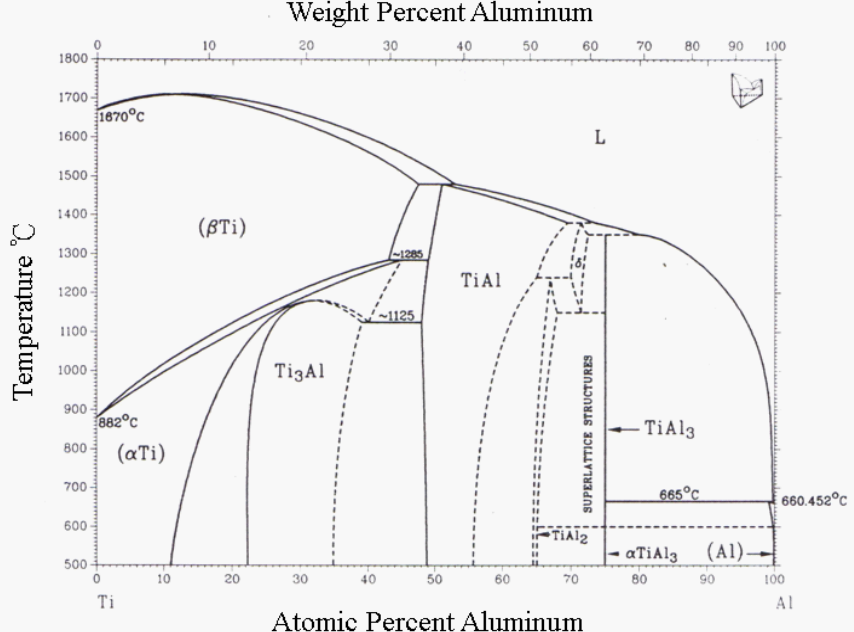

Figure 1(a)

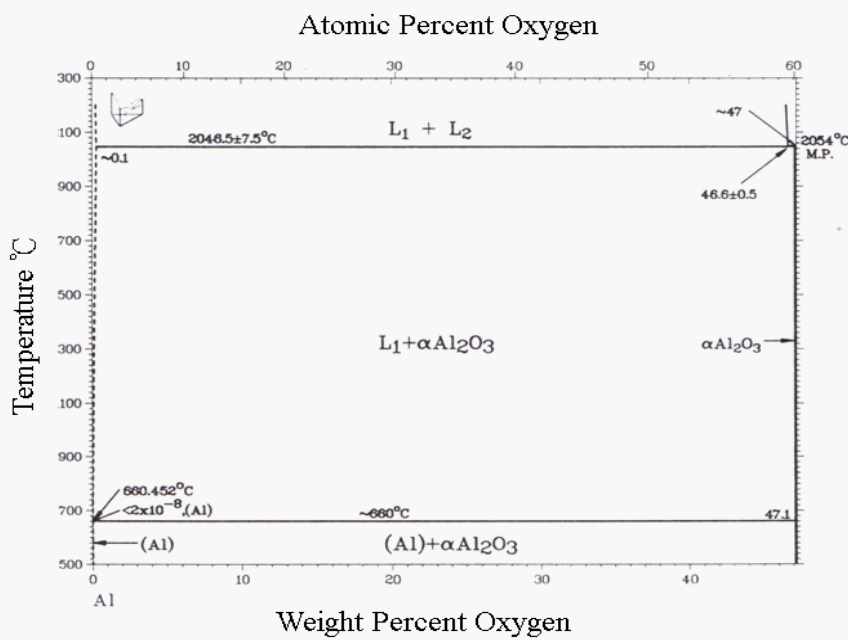

Figure 1(b)

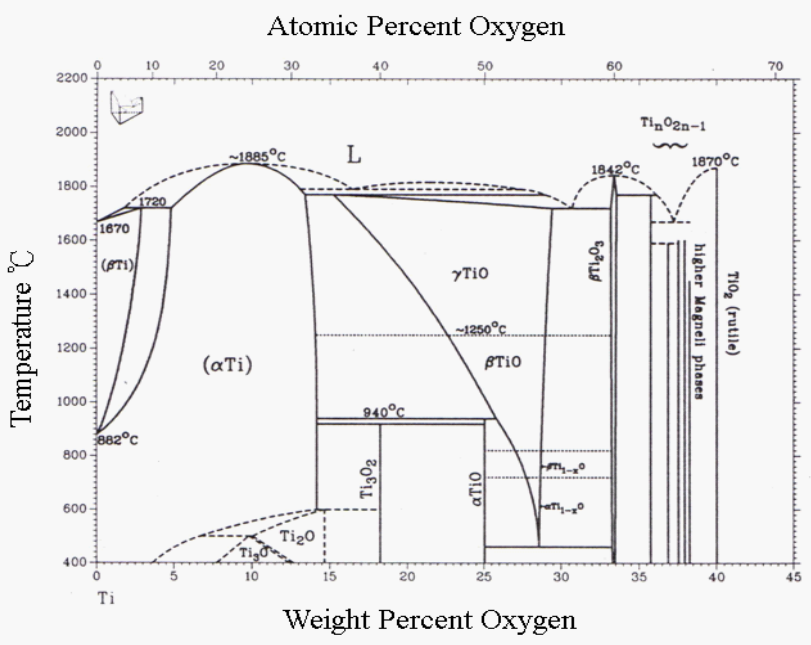

Figure 1(c)

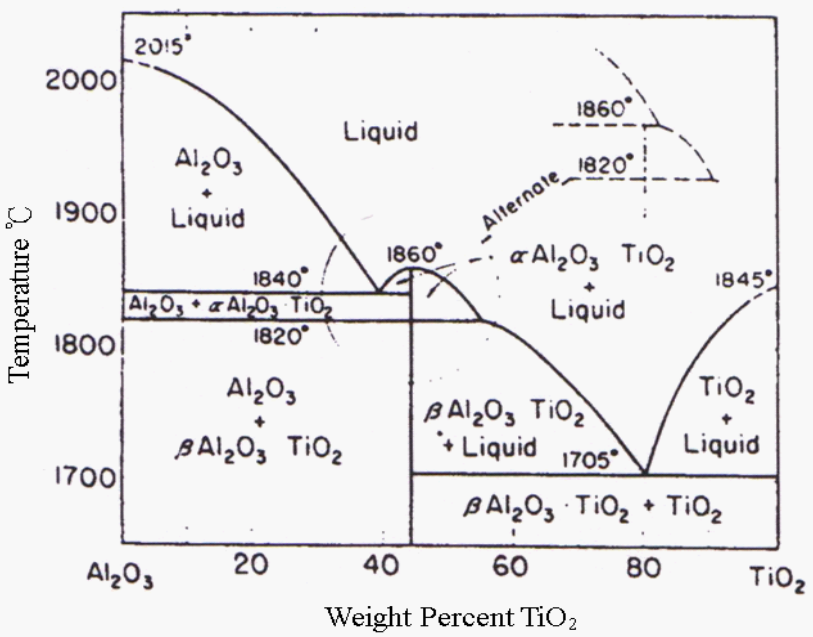

Figure 1(d)

Figure 1. Binary phase diagrams of (a) Ti-Al, (b) $\mathrm{Al}-\mathrm{O}$, (c) Ti-O, and (d) $\mathrm{Al}_{2} \mathrm{O}_{3}-\mathrm{TiO}_{2}$. (Wriedt, 1985; Caron et al., 1996; Copland, 2006).

Table 1. Twenty-three unstable phases can be neglected (deletion line) from the thirty-nine phases of the Ti-Al-O system in a temperature range of 1000 to $1200 \mathrm{~K}$.

\begin{tabular}{|c|c|c|c|c|c|c|}
\hline $\mathrm{Al}_{(\mathrm{s}, \mathrm{l})}$ & $A t_{(y)}$ & $\mathrm{Ti}_{(\alpha)}$ & $\mathrm{Ti}_{(\beta)}$ & $\mathrm{Fi}_{(\mathrm{tg})}$ & $\mathrm{O}_{2(\mathrm{~g})}$ & $\mathrm{Al}_{2} \mathrm{O}_{3(\alpha)}$ \\
\hline $\mathrm{Al}_{z} \Theta_{z(*)}$ & $A_{2} \theta_{3(\theta)}$ & $\mathrm{Al}_{2} \Theta_{3(H)}$ & $\mathrm{Al}_{z} \ominus_{\text {t(t) }}$ & $\mathrm{Al}_{2} \theta_{\text {(t) }}$ & $A \mid \theta_{\text {zt(5) }}$ & $A\left(\theta_{\text {tot }}\right.$ \\
\hline$F i \theta_{(t)}$ & $\mathrm{TiO}_{(\alpha)}$ & $F i \theta_{(A)}$ & $\mp i \theta_{(H)}$ & $\mathrm{TiO}_{2(\mathrm{~s})}$ & $\mathrm{Fi}_{z} \theta_{++1}$ & $\mathrm{Ti}_{2} \mathrm{O}_{3(\alpha)}$ \\
\hline $\mathrm{Fi}_{2} \theta_{3(\beta)}$ & $\mathrm{Fi}_{3} \Theta_{+}$ & $\mathrm{Fi}_{3} \Theta_{z+1}$ & $\mathrm{Ti}_{3} \mathrm{O}_{5(\alpha)}$ & $\mathrm{Ii}_{3} \theta_{5(\beta)}$ & $\mathrm{Ti}_{4} \mathrm{O}_{7(\mathrm{~s})}$ & $\mathrm{Fi}_{5} \Theta_{\text {Af }}$ \\
\hline $\mathrm{Fi}_{6} \ominus_{1+\text { (f) }}$ & $F_{i} \theta_{17(+1)}$ & $F_{i_{16}} \theta_{3(+\infty)}$ & 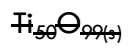 & $\operatorname{TiAl}_{(\mathrm{s})}$ & $\mathrm{TiAl}_{2(\mathrm{~s})}$ & $\mathrm{TiAl}_{3(\mathrm{~s})}$ \\
\hline $\mathrm{Fi}_{8} \Theta_{+5(+)}$ & $\mathrm{Ti}_{2} \mathrm{Al}_{5(\mathrm{~s})}$ & $\mathrm{Ti}_{3} \mathrm{Al}_{(\mathrm{s})}$ & $\mathrm{TiAl}_{2} \mathrm{O}_{5(\mathrm{~s})}$ & & & \\
\hline
\end{tabular}




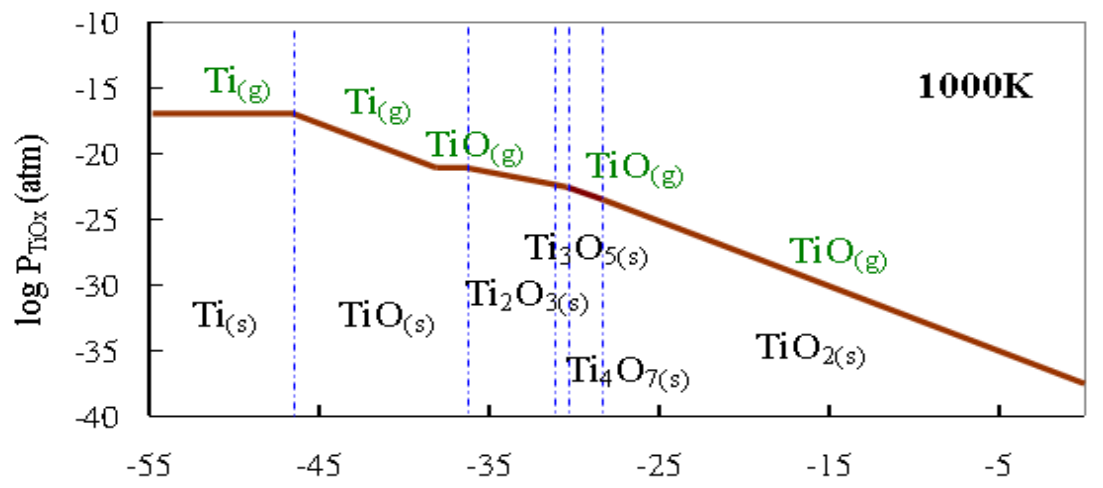

(a)

$\log \mathrm{P}_{\mathrm{O} 2}(\mathrm{~atm})$

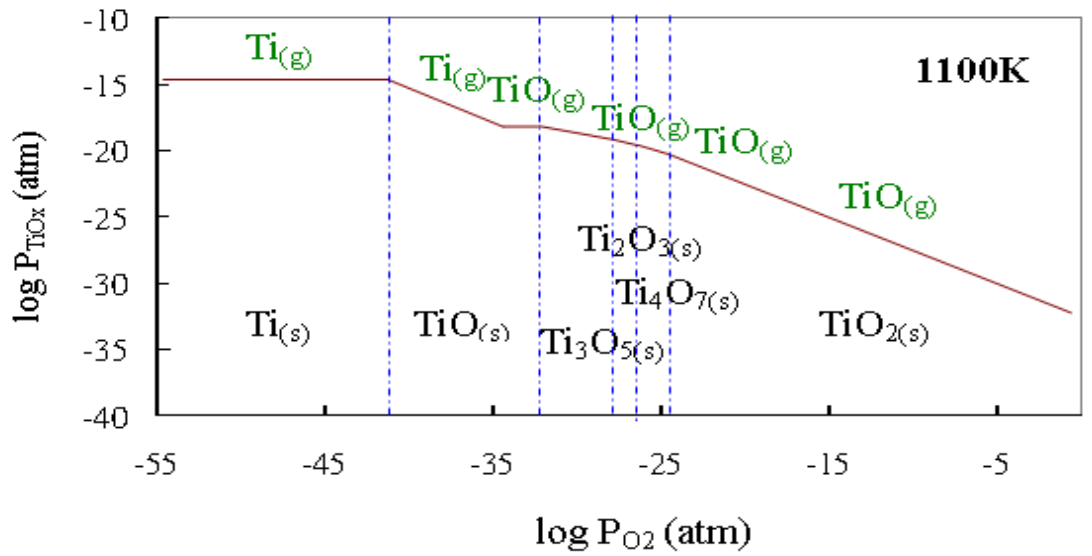

(b)

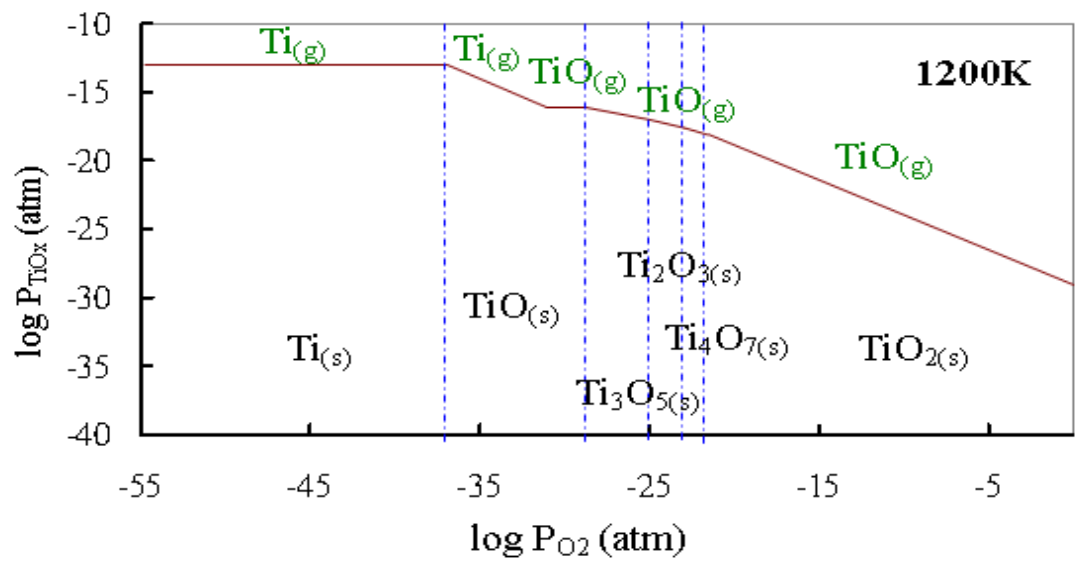

(c)

Figure 2. The pressure curves of $\mathrm{Ti}_{(\mathrm{g})}$ and $\mathrm{TiO}_{(\mathrm{g})}$ in $\mathrm{Ti}(\mathrm{s}), \mathrm{TiO}_{(\mathrm{s}){ }^{\prime}} \mathrm{Ti}_{2} \mathrm{O}_{3(\mathrm{~s}))^{\prime}} \mathrm{Ti}_{3} \mathrm{O}_{5(\mathrm{~s})^{\prime}} \mathrm{Ti}_{4} \mathrm{O}_{7(\mathrm{~s})^{\prime}}$ and $\mathrm{TiO}_{2(\mathrm{~s})}$ condensed phases at (a) $1000 \mathrm{~K}$, (b) $1100 \mathrm{~K}$, and (c) $1200 \mathrm{~K}$. 


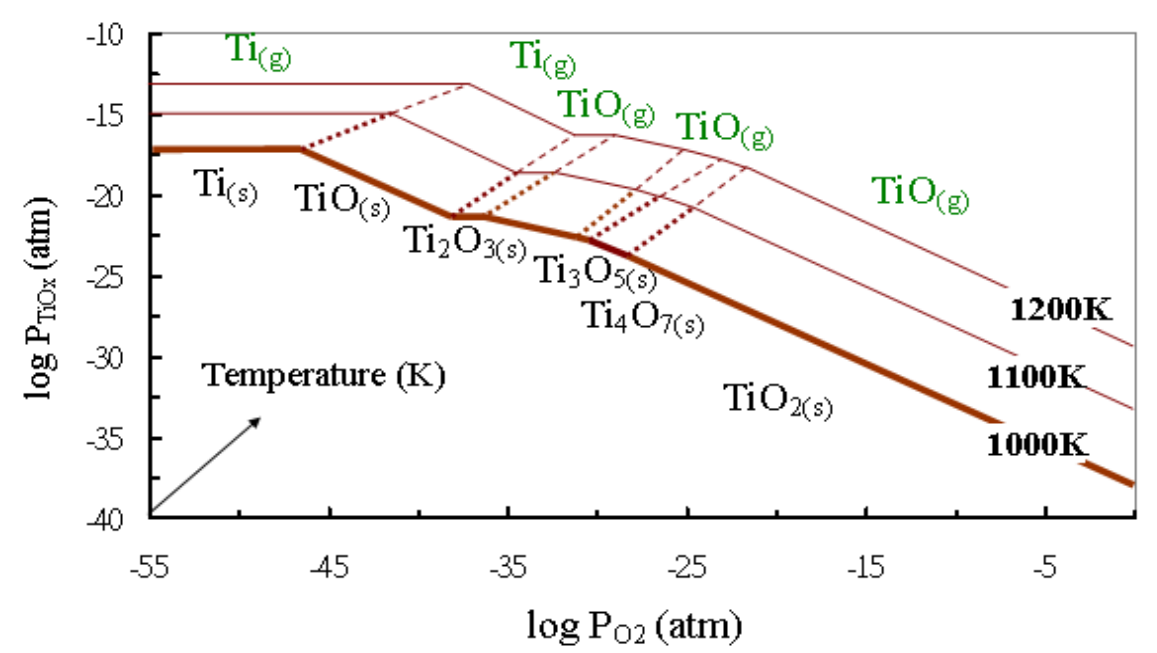

(a)

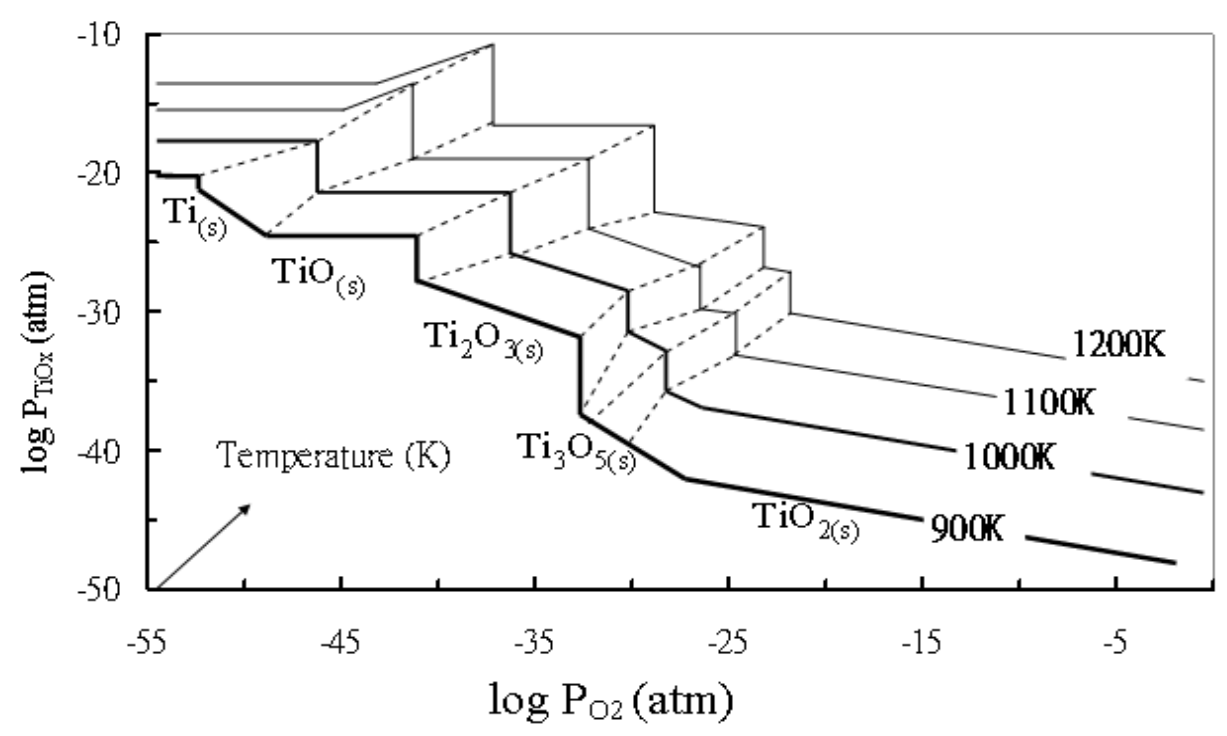

(b)

Figure 3. Belted and stepped curves of $\mathrm{Ti}_{(\mathrm{g})}$ and $\mathrm{TiO}_{(\mathrm{g})}$ dominating the condensed phases of $\mathrm{Ti}_{(\mathrm{s}))^{\prime}} \mathrm{TiO}_{(\mathrm{s})^{\prime}} \mathrm{Ti}_{3} \mathrm{O}_{5(\mathrm{~s})^{\prime}}$ $\left.\mathrm{Ti}_{4} \mathrm{O}_{7(\mathrm{~s})}\right)^{\prime}$ and $\mathrm{TiO}_{2(\mathrm{~s})}$ at temperatures of 900 to $1200 \mathrm{~K}$.

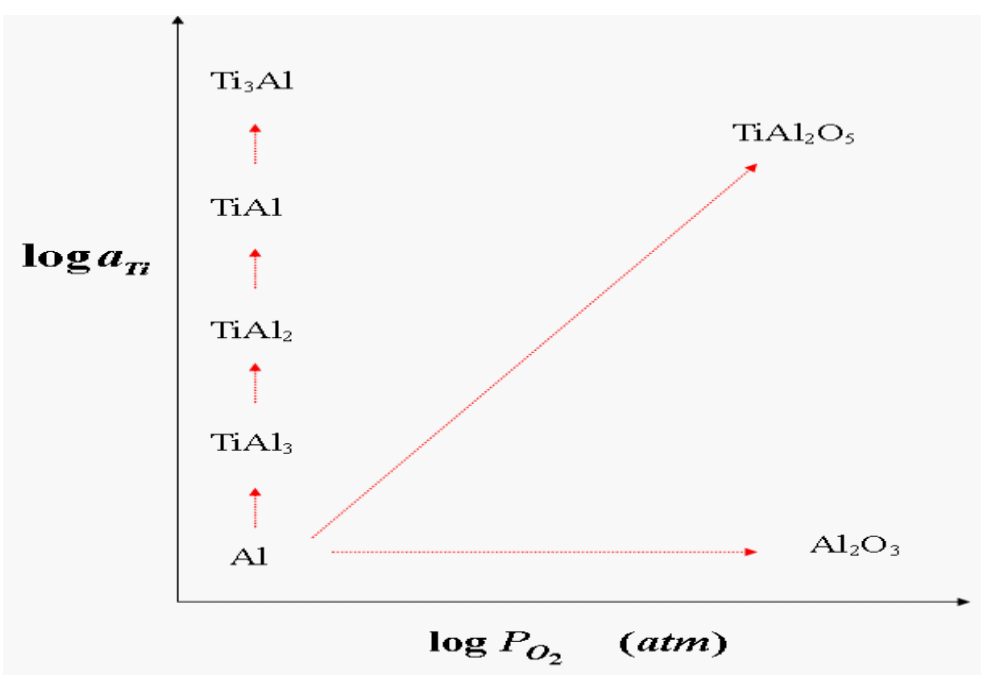

Figure 4

Figure 4. Schematic of Ti-Al-O thermodynamic system in the isothermal stability diagram. 

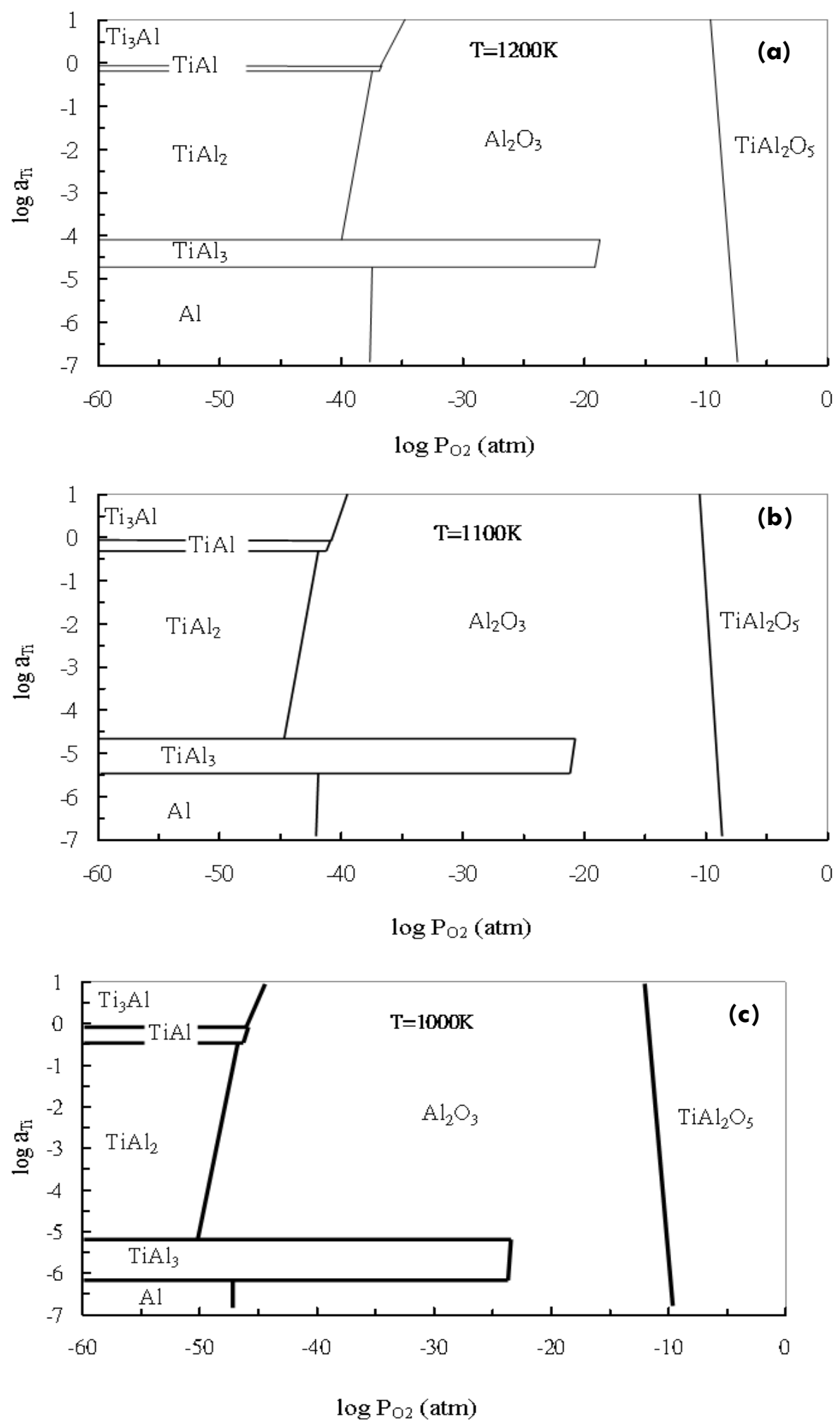

Figure 5. Condensed phases of Ti-Al-O compound regions on the $\log a_{\mathrm{TiO} 2}-\log P_{\mathrm{O} 2}$ diagram in Ti$\mathrm{Al}-\mathrm{O}$ isothermal stability diagram at (a) $1000 \mathrm{~K}$, (b) $1100 \mathrm{~K}$, and (c) $1200 \mathrm{~K}$. 
Table 2. Reactions, equilibrium constant $(K)$, and formation-Gibbs free energies $\left(\Delta G_{f}\right)$ of twelve possible compounds forming at Ti-Ai interface under oxygen pressure at a temperature range of 1000 to $1200 \mathrm{~K}$.

\begin{tabular}{|c|c|c|c|}
\hline No. & Eqn. Reactions & $\log K$ & $\Delta G f(\mathrm{~kJ} / \mathrm{mol})$ \\
\hline 1 & $\mathrm{Ti}_{(\mathrm{s})}+\frac{1}{2} \mathrm{O}_{2(\mathrm{~g})} \rightarrow \mathrm{TiO}_{(\mathrm{s})}$ & $-4.97+\frac{28262.2}{T}$ & $-541.1+0.09 T$ \\
\hline 2 & $2 \mathrm{Ti}_{(\mathrm{s})}+\frac{3}{2} \mathrm{O}_{2(\mathrm{~g})} \rightarrow \mathrm{Ti}_{2} \mathrm{O}_{3(\mathrm{~s})}$ & $-14.14+\frac{78926.6}{T}$ & $-1511.2+0.271 T$ \\
\hline 3 & $3 \mathrm{Ti}_{(\mathrm{s})}+\frac{5}{2} \mathrm{O}_{2(\mathrm{~g})} \rightarrow \mathrm{Ti}_{3} \mathrm{O}_{5(\mathrm{~s})}$ & $-23.72+\frac{127944.3}{T}$ & $-2449.7+0.452 T$ \\
\hline 4 & $4 \mathrm{Ti}_{(\mathrm{s})}+\frac{7}{2} \mathrm{O}_{2(\mathrm{~g})} \rightarrow \mathrm{Ti}_{4} \mathrm{O}_{7(\mathrm{~s})}$ & $-32.16+\frac{177194.3}{T}$ & $-3392.7+0.621 T$ \\
\hline 5 & $\mathrm{Ti}_{(\mathrm{s})}+\mathrm{O}_{2(\mathrm{~g})} \rightarrow \mathrm{TiO}_{2(\mathrm{~s})}$ & $-9.28+\frac{49113.4}{T}$ & $-940.4+0.181 T$ \\
\hline 6 & $\mathrm{Ti}_{(\mathrm{s})}+2 \mathrm{Al}_{(\mathrm{l})}+\frac{5}{2} \mathrm{O}_{2(\mathrm{~g})} \rightarrow \mathrm{TiAl}_{2} \mathrm{O}_{5(\mathrm{~s})}$ & $-26.0+\frac{137873.5}{T}$ & $-2640.1+0.500 T$ \\
\hline 7 & $2 \mathrm{Al}_{(\mathrm{l})}+\frac{3}{2} \mathrm{O}_{2(\mathrm{~g})} \rightarrow \mathrm{Al}_{2} \mathrm{O}_{3(\mathrm{~s})}$ & $-17.29+\frac{88395.7}{T}$ & $-1692.5+0.33 T$ \\
\hline 8 & $3 \mathrm{Ti}_{(\mathrm{s})}+\mathrm{Al}_{(\mathrm{l})} \rightarrow \mathrm{Ti}_{3} \mathrm{Al}_{(\mathrm{s})}$ & $-0.35+\frac{1547.7}{T}$ & $-29.6+0.007 T$ \\
\hline 9 & $\mathrm{Ti}_{(\mathrm{s})}+\mathrm{Al}_{(\mathrm{l})} \rightarrow \mathrm{TiAl}_{(\mathrm{s})}$ & $-0.88+\frac{1955.7}{T}$ & $-37.5+0.017 T$ \\
\hline 10 & $\mathrm{Ti}_{(\mathrm{s})}+2 \mathrm{Al}_{(\mathrm{l})} \rightarrow \mathrm{TiAl}_{2(\mathrm{~s})}$ & $-0.58+\frac{2290.6}{T}$ & $-43.9+0.011 T$ \\
\hline 11 & $2 \mathrm{Ti}_{(\mathrm{s})}+5 \mathrm{Al}_{(\mathrm{l})} \rightarrow \mathrm{Ti}_{2} \mathrm{Al}_{5(\mathrm{~s})}$ & $\log K=-2.87+\frac{3797}{T}$ & $-34.4+0.055 T$ \\
\hline 12 & $\mathrm{Ti}_{(\mathrm{s})}+3 \mathrm{Al}_{(\mathrm{l})} \rightarrow \mathrm{TiAl}_{3(\mathrm{~s})}$ & $\log K=-1.33+\frac{9653}{T}$ & $-40.3+0.010 T$ \\
\hline
\end{tabular}


Table 3. Eleven coexistence terms of reaction and Gibbs free energies of Ti-Al-O system on bivariant equilibria lines in a temperature range of 1000 to $1200 \mathrm{~K}$.

\begin{tabular}{|c|c|c|c|c|}
\hline No. & Interface & Reaction & $\log K$ & Equation \\
\hline 1 & $\mathrm{Al} / \mathrm{Al}_{2} \mathrm{O}_{3}$ & $2 \mathrm{Al}_{(\mathrm{l})}+\frac{3}{2} \mathrm{O}_{2(\mathrm{~g})} \rightarrow \mathrm{Al}_{2} \mathrm{O}_{3(\alpha)}$ & $-17.29+\frac{88396}{T}$ & $\log P_{O_{2}}=\frac{-2}{3} \log K$ \\
\hline 2 & $\mathrm{Al} / \mathrm{TiAl}_{3}$ & $3 \mathrm{Al}_{(\mathrm{l})}+\mathrm{Ti} \rightarrow \mathrm{TiAl}_{3(\mathrm{~s})}$ & $-1.33+\frac{9653}{T}$ & $\log a_{\mathrm{Ti}}=-\log K$ \\
\hline 3 & $\mathrm{TiAl}_{3} / \mathrm{Ti}_{2} \mathrm{Al}_{5}$ & $\frac{5}{3} \mathrm{TiAl}_{3(\mathrm{~s})}+\frac{1}{3} \mathrm{Ti} \rightarrow \mathrm{Ti}_{2} \mathrm{Al}_{5(\mathrm{~s})}$ & $4.68-\frac{2265}{T}$ & $\log a_{\mathrm{Ti}}=-3 \log K$ \\
\hline 4 & $\mathrm{Ti}_{2} \mathrm{Al}_{5} / \mathrm{TiAl}_{2}$ & $\frac{5}{2} \mathrm{TiAl}_{2(\mathrm{~s})} \rightarrow \frac{1}{2} \mathrm{Ti}+\mathrm{Ti}_{2} \mathrm{Al}_{5(\mathrm{~s})}$ & $-1.42-\frac{1930}{T}$ & $\log a_{\mathrm{Ti}}=2 \log K$ \\
\hline 5 & $\mathrm{TiAl}_{2} /$ TiAl & $2 \mathrm{TiAl}_{(\mathrm{s})} \rightarrow \mathrm{TiAl}_{2(\mathrm{~s})}+\mathrm{Ti}$ & $1.18-\frac{1621}{T}$ & $\log a_{\mathrm{Ti}}=\log K$ \\
\hline 6 & $\mathrm{TiAl} / \mathrm{Ti}_{3} \mathrm{Al}$ & $\mathrm{TiAl}_{(s)}+2 \mathrm{Ti} \rightarrow \mathrm{Ti}_{3} \mathrm{Al}_{(\mathrm{s})}$ & $0.527-\frac{408}{T}$ & $\log a_{\mathrm{Ti}}=\frac{-1}{2} \log K$ \\
\hline 7 & $\mathrm{Al}_{2} \mathrm{O}_{3} / \mathrm{TiAl}_{3}$ & $\frac{3}{2} \mathrm{Al}_{2} \mathrm{O}_{3(\alpha \alpha}+\mathrm{Ti} \rightarrow \frac{9}{2} \mathrm{O}_{2(\mathrm{~g})}+\mathrm{TiAl}_{3(\mathrm{~s})}$ & $22.6-\frac{122941}{T}$ & $\log P_{\mathrm{O}_{2}}=\frac{2}{9}\left(\log K+\log a_{\mathrm{Ti}}\right)$ \\
\hline 8 & $\mathrm{Al}_{2} \mathrm{O}_{3} / \mathrm{TiAl}_{2}$ & $\mathrm{Al}_{2} \mathrm{O}_{3(\alpha \alpha}+\mathrm{Ti} \rightarrow \frac{3}{2} \mathrm{O}_{2(\mathrm{~g})}+\mathrm{TiAl}_{2(\mathrm{~s})}$ & $16.15-\frac{86440}{T}$ & $\log P_{\mathrm{O}_{2}}=\frac{2}{3}\left(\log a_{\mathrm{Ti}}+\log K\right)$ \\
\hline 9 & $\mathrm{Al}_{2} \mathrm{O}_{3} / \mathrm{TiAl}$ & $\frac{1}{2} \mathrm{Al}_{2} \mathrm{O}_{3(\alpha \alpha}+\mathrm{Ti} \rightarrow \frac{3}{4} \mathrm{O}_{2(\mathrm{~g})}+\mathrm{TiAl}_{(\mathrm{s})}$ & $7.77-\frac{42242}{T}$ & $\log P_{\mathrm{O}_{2}}=\frac{4}{3}\left(\log K+\log a_{\mathrm{Ti}}\right)$ \\
\hline 10 & $\mathrm{Al}_{2} \mathrm{O}_{3} / \mathrm{Ti}_{3} \mathrm{Al}$ & $\frac{1}{2} \mathrm{Al}_{2} \mathrm{O}_{3(\alpha)}+3 \mathrm{Ti} \rightarrow \frac{3}{4} \mathrm{O}_{2(g)}+\mathrm{Ti}_{3} \mathrm{Al}_{(s)}$ & $8.12-\frac{42650}{T}$ & $\log P_{\mathrm{O}_{2}}=\frac{4}{3}\left(\log K+3 \log a_{\mathrm{Ti}}\right)$ \\
\hline 11 & $\mathrm{Al}_{2} \mathrm{O}_{3} / \mathrm{TiAl}_{2} \mathrm{O}_{5}$ & $\mathrm{Al}_{2} \mathrm{O}_{3(\alpha)}+\mathrm{Ti}+\frac{7}{2} \mathrm{O}_{2(\mathrm{~g})} \rightarrow \mathrm{TiAl}_{2} \mathrm{O}_{5(\mathrm{~s})}$ & $-8.71+\frac{49478}{T}$ & $\log P_{\mathrm{O}_{2}}=\frac{-2}{7}\left(\log K+\log a_{\mathrm{T}_{\mathrm{i}}}\right)$ \\
\hline
\end{tabular}

The relationships between the vapor pressures and are:

$$
\begin{aligned}
& \log K_{2}=\log P_{\mathrm{Ti}} \\
& \log K_{3}=\log P_{\mathrm{TiO}} \\
& \log K_{4}=3 \log P_{\mathrm{TiO}}+\log P_{\mathrm{O}_{2}} \\
& \log K_{5}=4 \log P_{\mathrm{TiO}}+\frac{3}{2} \log P_{\mathrm{O}_{2}} \\
& \log K_{6}=\log P_{\mathrm{TiO}}+\frac{1}{2} \log P_{\mathrm{O}_{2}}
\end{aligned}
$$

The partial pressure of $\mathrm{Ti}_{(\mathrm{g})}$ and $\mathrm{TiO}_{(\mathrm{g})}$ dominates the condensed phases $\mathrm{Ti}_{(\mathrm{s})^{\prime}}, \mathrm{TiO}_{(s)^{\prime}}, \mathrm{Ti}_{3} \mathrm{O}_{5(\mathrm{~s})^{\prime}}, \mathrm{ii}_{4} \mathrm{O}_{7(\mathrm{~s})^{\prime}}$ and $\mathrm{TiO}_{2(s)^{\prime}}$ as shown in Figure 2. Figures $3(\mathrm{a})$ and $3(\mathrm{~b})$ also show that belted and stepped curves of $\mathrm{Ti}_{(\mathrm{g})}$ and $\mathrm{TiO}_{(\mathrm{g})}$ dominate the condensed phases $\mathrm{Ti}_{(\mathrm{s})}$ $\left.\mathrm{TiO}_{(\mathrm{s})^{\prime}} \mathrm{Ti}_{3} \mathrm{O}_{5(\mathrm{~s})}\right)^{\prime} \mathrm{Ti}_{4} \mathrm{O}_{7\left(\mathrm{~s}^{\prime}\right.}$ and $\mathrm{TiO}_{2(\mathrm{~s})}$ in a temperature range of 900 to $1200 \mathrm{~K}$, based on the results in Table 2.

\section{Condensed Phases}

Seven condensed phases $\left(\mathrm{Al}_{\left.()^{\prime}\right)^{\prime}} \mathrm{TiAl}_{3(\mathrm{~s})^{\prime}}, \mathrm{Ti}_{2} \mathrm{Al}_{5(\mathrm{~s})^{\prime}}, \mathrm{TiAl}_{2(\mathrm{~s})^{\prime}}, \mathrm{TiAl}_{(\mathrm{s})^{\prime}}\right.$ $\mathrm{Ti}_{3} \mathrm{Al}_{(s)^{\prime}}$ and $\left.\mathrm{Al}_{2} \mathrm{O}_{3(\alpha)}\right)$ can be arranged on a $\left(\log P_{\mathrm{O} 2}-\log \mathrm{a}_{\mathrm{Ti}}\right)$ diagram of the Ti-Al-O system at constant temperature. Figure 4 depicts the regions of each condensed phase along the axis in the $\left(\log P_{\mathrm{O}_{2}}-\log a_{\mathrm{Ti}}\right)$ diagram. This so-called isothermal stability diagram is useful in estimating the relationships between each pair of condensed phases in the Ti-Al-O system; however, the interfaces between each pair of phases cannot be clearly determined from the schematic diagram. Since multiple phases can accumulate at an interface, 35 assemblies can possibly be constructed in the $\mathrm{O}_{(\mathrm{g})^{\prime}} \mathrm{Ti}_{(\mathrm{s})^{\prime}}, \mathrm{Al}_{\left.()^{\prime}\right)^{\prime}}, \mathrm{TiAl}_{3(\mathrm{~s})^{\prime}}, \mathrm{TiAl}_{2(\mathrm{~s})}, \mathrm{TiAl}_{(\mathrm{s})^{\prime}}, \mathrm{TiAl}_{2} \mathrm{O}_{5^{\prime}}, \mathrm{Ti}_{3} \mathrm{Al}_{(\mathrm{s})^{\prime}}$ and $\mathrm{Al}_{2} \mathrm{O}_{3}$ phases, namely $\mathrm{Al} / \mathrm{Al}_{2} \mathrm{O}_{3}, \mathrm{Al} / \mathrm{TiO}_{2}, \mathrm{Al} / \mathrm{TiAl}, \mathrm{Al} / \mathrm{TiAl}{ }_{2}$ $\mathrm{Al} / \mathrm{TiAl}_{3}, \mathrm{Al} / \mathrm{Ti}_{2} \mathrm{Al}_{5}, \mathrm{Al} / \mathrm{Ti}_{3} \mathrm{Al}, \mathrm{Al} / \mathrm{TiAl}_{2} \mathrm{O}_{5}, \mathrm{Al}_{2} \mathrm{O}_{3} / \mathrm{TiO}_{2}, \mathrm{Al}_{2} \mathrm{O}_{3} /$ $\mathrm{TiAl}, \mathrm{Al}_{2} \mathrm{O}_{3} / \mathrm{TiAl}_{2}, \mathrm{Al}_{2} \mathrm{O}_{3} / \mathrm{TiAl}_{3}, \mathrm{Al}_{2} \mathrm{O}_{3} / \mathrm{Ti}_{2} \mathrm{Al}_{5}, \mathrm{Al}_{2} \mathrm{O}_{3} / \mathrm{Ti}_{3} \mathrm{Al}, \mathrm{Al}_{2} \mathrm{O}_{3} /$ $\mathrm{TiAl}_{2} \mathrm{O}_{5}, \mathrm{TiO}_{2} / \mathrm{TiAl}, \mathrm{TiO}_{2} / \mathrm{TiAl}_{2}, \mathrm{TiO}_{2} / \mathrm{TiAl}_{3}, \mathrm{TiO}_{2} / \mathrm{Ti}_{2} \mathrm{Al}_{5}, \mathrm{TiO}_{2} /$ $\mathrm{Ti}_{3} \mathrm{Al}, \mathrm{TiO}_{2} / \mathrm{TiAl}_{2} \mathrm{O}_{5}, \mathrm{TiAl} / \mathrm{TiAl}_{2}, \mathrm{TiAl} / \mathrm{TiAl}_{3}, \mathrm{TiAl} / \mathrm{Ti}_{2} \mathrm{Al}_{5}, \mathrm{TiAl} / \mathrm{Ti}_{3} \mathrm{Al}$, $\mathrm{TiAl} / \mathrm{TiAl}_{2} \mathrm{O}_{5}, \mathrm{TiAl}_{2} / \mathrm{TiAl}_{3}, \mathrm{TiAl}_{2} / \mathrm{Ti}_{2} \mathrm{Al}_{5}, \mathrm{TiAl}_{2} / \mathrm{Ti}_{3} \mathrm{Al}, \mathrm{TiAl}_{2} / \mathrm{TiAl}_{2} \mathrm{O}_{5}$ $\mathrm{Ti}_{2} \mathrm{Al}_{5} / \mathrm{TiAl}_{2} \mathrm{O}_{5}, \mathrm{Ti}_{2} \mathrm{Al}_{5} / \mathrm{Ti}_{3} \mathrm{Al}, \mathrm{TiAl}_{3} / \mathrm{Ti}_{3} \mathrm{Al}, \mathrm{TiAl}_{3} / \mathrm{TiAl}_{2} \mathrm{O}_{5}$, and $\mathrm{Ti}_{3} \mathrm{Al} /$ $\mathrm{TiAl}_{2} \mathrm{O}_{5}$.

However, as shown in Figure 4, no interfaces of $\mathrm{Al} / \mathrm{TiAl}_{3}, \mathrm{Al} /$ $\mathrm{TiAl}, \mathrm{Al} / \mathrm{Ti}_{3} \mathrm{Al}, \mathrm{TiAl}_{3} / \mathrm{TiAl}_{2}, \mathrm{TiAl}_{3} / \mathrm{TiAl}, \mathrm{TiAl}_{3} / \mathrm{Ti}_{3} \mathrm{Al}$, or $\mathrm{TiAl}_{2} / \mathrm{Ti}_{3} \mathrm{Al}$ are found. Therefore, there are 35 terms, except those associated with these ten non-existing interfaces, are present, as shown in Figure 4. Nevertheless, there is still a problem of overlapping 


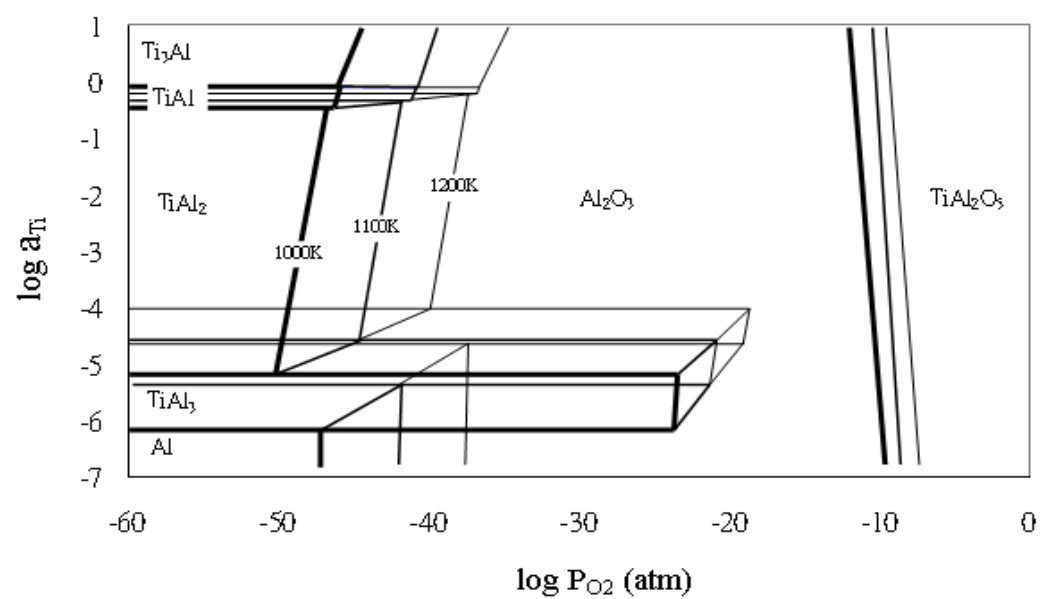

Figure 6. Isothermal stability diagram of Ti-Al-O compounds in a temperature range of 1000 to $1200 \mathrm{~K}$ under various oxygen pressures and Ti activity values.

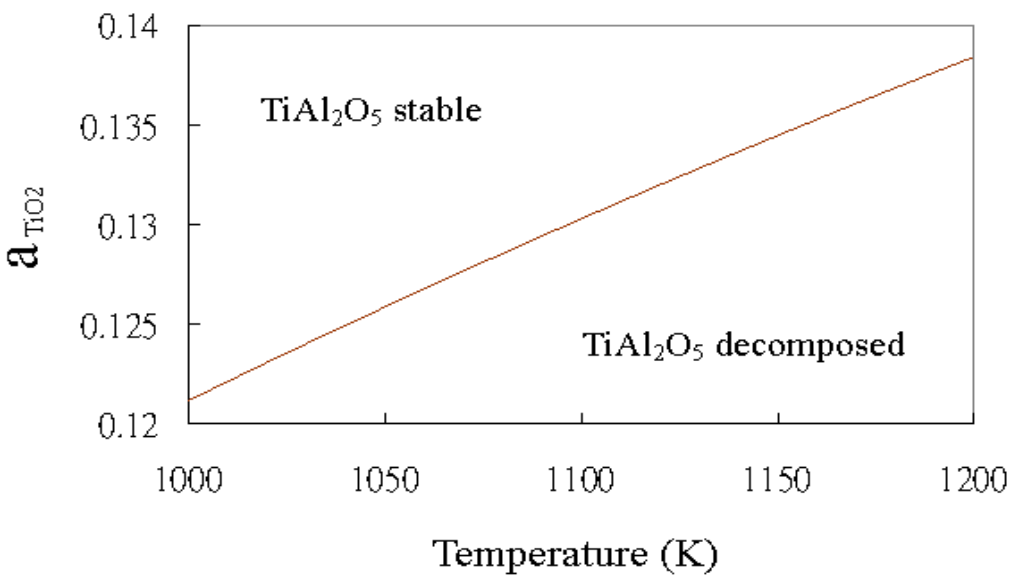

Figure 7. Decomposition curve of spinel $\left(\mathrm{TiAl}_{2} \mathrm{O}_{5}\right)$ with varying $\mathrm{TiO}_{2}$ activity from 1000 to $1200 \mathrm{~K}$.

in the $\mathrm{Al}_{2} \mathrm{O}_{3}$ and $\mathrm{TiAl}_{2} \mathrm{O}_{5}$ regions. Thus, several other interfaces should not exist and therefore should be removed from the 35 terms. The interfaces $\mathrm{Al} / \mathrm{TiAl}_{2} \mathrm{O}_{5}, \mathrm{Al} / \mathrm{TiAl}_{3}, \mathrm{Al} / \mathrm{TiAl}_{2}, \mathrm{Al} / \mathrm{TiAl}$, and $\mathrm{Al} / \mathrm{Ti}_{3} \mathrm{Al}$ must be omitted to label the compound's domains in Figure 4. Finally, only ten coexisting terms were retained from the original terms. Table 3 presents the equations and obtained Gibbs free energy of the ten coexistent terms. Combining Figure 4 with the thermodynamic data from Table 3 yields the interfaces of compounds, as presented in Figure 5.

For example, to estimate the boundaries of the TiAl region, firstly, reactions 15, 16, 17, and 18, and their Gibbs free energy of formation are obtained from Table 2. Secondly, the relationship between the activity of $\mathrm{Ti}$ and the oxygen partial pressure is obtained from reaction 15, under the $\mathrm{TiAl} / \mathrm{Al}_{2} \mathrm{O}_{3}$ coexistence condition. Thirdly, the activity of $\mathrm{Ti}$ is calculated from reaction 16 under the $\mathrm{TiAl} / \mathrm{TiAl}_{2}$ coexistence condition. Finally, reaction 17 can also be used to calculate the activity of $\mathrm{Ti}$ under the TiAl/ $\mathrm{Ti}_{3} \mathrm{Al}$ coexistence condition,

$$
\begin{aligned}
& \frac{1}{2} \mathrm{Al}_{2} \mathrm{O}_{3(\alpha)}+\mathrm{Ti}_{(\mathrm{s})} \rightarrow \frac{3}{4} \mathrm{O}_{2(\mathrm{~g})}+\mathrm{TiAl}_{(\mathrm{s})} \\
& 2 \mathrm{TiAl}_{(\mathrm{s})} \rightarrow \mathrm{TiAl}_{2(\mathrm{~s})}+\mathrm{Ti}_{(\mathrm{s})}
\end{aligned}
$$

$$
\mathrm{TiAl}_{(\mathrm{s})}+2 \mathrm{Ti}_{(\mathrm{s})} \rightarrow \mathrm{Ti}_{3} \mathrm{Al}_{(\mathrm{s})}
$$

Additionally, the oxygen pressure obtained from reaction 15 is:

$$
\log P_{\mathrm{O}_{2}}=\frac{4}{3}\left(\log K+\log a_{\mathrm{Ti}}\right)
$$

The oblique line in Figure 5 drawn along the axis in the $\log P_{\mathrm{O} 2}$ - $\log a_{T i}$ diagram represents the interface between $\mathrm{Al}_{2} \mathrm{O}_{3}$ and TiAl. The activity of Ti between $\mathrm{TiAl}$ and $\mathrm{TiAl}_{2}$ can be determined from reaction 16 as follows:

$$
\log a_{\mathrm{Ti}}=\log K
$$

Therefore, in Figure 5, one horizontal line (the interface between $\mathrm{TiAl}$ and $\mathrm{TiAl}_{2}$ ) is determined by reaction 19, and the other horizontal line (the interface between $\mathrm{TiAl}$ and $\mathrm{Ti}_{3} \mathrm{Al}$ ) is determined from reaction 17; the three lines define the TiAl region. Accordingly, in Figure 5, the regions associated with the other compounds can also be determined, and then the equations of univariant equilibria (triple-phase coexistence) points can be calculated, as shown in Table 3. Furthermore, overlapping Figures $5(a),(b)$, and (c) produces a 3-D (axes temperature, log $\mathrm{P}_{\mathrm{O} 2}-\log \mathrm{a}_{\mathrm{Ti}}$ ) isothermal stability diagram, as presented in Figure 


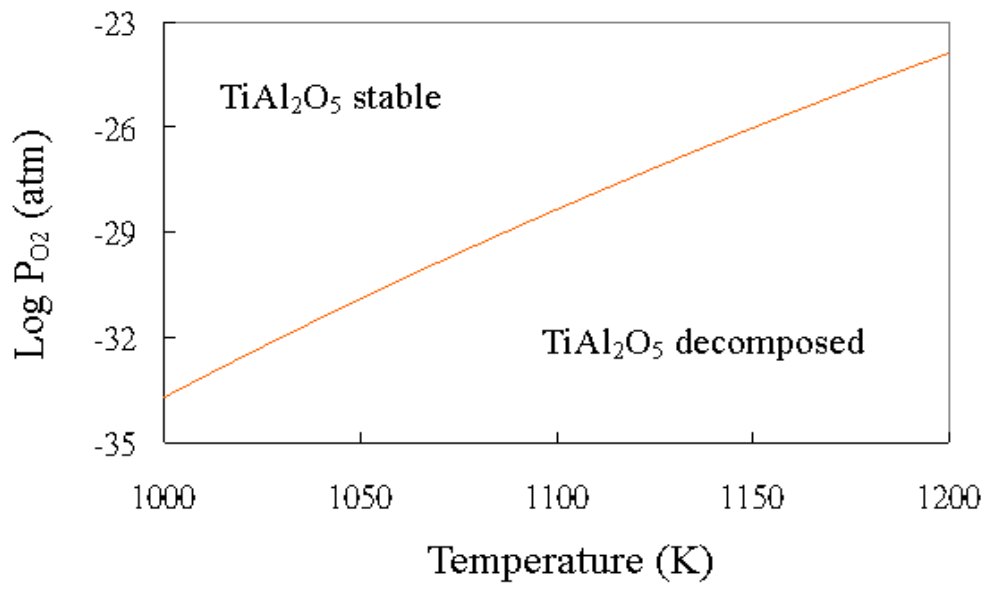

Figure 8. Decomposition curve of spinel $\left(\mathrm{TiAl}_{2} \mathrm{O}_{5}\right)$ with varying partial pressure of oxygen from 1000 to $1200 \mathrm{~K}$.

6. The temperature range is widened from 1000 to $1200 \mathrm{~K}$. The diagram clearly shows regions for $\mathrm{Al}, \mathrm{Al}_{2} \mathrm{O}_{3}, \mathrm{TiAl}_{3^{\prime}}, \mathrm{TiAl}_{3^{\prime}}, \mathrm{Ti}_{3} \mathrm{Al}_{5^{\prime}}$ $\mathrm{TiAl}, \mathrm{Ti}_{3} \mathrm{Al}$, and $\mathrm{TiAl}_{2} \mathrm{O}_{5}$ at various temperatures.

\section{Discussion}

\section{Sorting Compounds by Gibbs Free Energy of Formation}

The thermodynamic stabilities of compounds in a Ti-Al-O system were evaluated by considering their Gibbs free energy, based on the data in Table 2. In a Ti-Al-O system, in ascending order of Gibbs free energy, the compounds at $1000 \mathrm{~K}$ are: $\mathrm{Ti}_{4} \mathrm{O}_{7(\mathrm{~s})} \rightarrow \mathrm{TiAl}_{2} \mathrm{O}_{5(\mathrm{~s})} \rightarrow \mathrm{Ti}_{3} \mathrm{O}_{5(\alpha)} \rightarrow \mathrm{Al}_{2} \mathrm{O}_{3(\alpha)} \rightarrow \mathrm{Ti}_{2} \mathrm{O}_{3(\alpha)} \rightarrow \mathrm{TiO}_{2(s)}$ $\rightarrow \mathrm{TiO}_{(\alpha)} \rightarrow \mathrm{Ti}_{3} \mathrm{Al}_{(\mathrm{s})} \rightarrow \mathrm{TiAl}_{2(\mathrm{~s})} \rightarrow \mathrm{TiAl}_{3(\mathrm{~s})} \rightarrow \mathrm{Ti}_{2} \mathrm{Al}_{5(\mathrm{~s})} \rightarrow \mathrm{TiAl}_{(\mathrm{s})}$. Thus, $\mathrm{Ti}_{4} \mathrm{O}_{7(\mathrm{~s})}$ is the most stable phase, and $\mathrm{TiAl}(\mathrm{s})$ is the least stable compound. In conclusion, the order of formation of Ti-Al-O intermetallic compounds can be determined from thermodynamics. The Ti3Al(s) phase is the first to appear, but the TiAl phase is the last to appear.

\section{Equations for Spinel Formation}

Four different equations for the formation of spinel are,

$$
\begin{aligned}
& \mathrm{TiO}_{2(\mathrm{~s})}+\mathrm{Al}_{2} \mathrm{O}_{3(\alpha)} \rightarrow \mathrm{TiAl}_{2} \mathrm{O}_{5(\mathrm{~s})}, \quad(\Delta G=-7.2-0.011 T(\mathrm{~kJ} / \mathrm{mol})) \\
& \mathrm{Ti}_{(\mathrm{s})}+\frac{5}{3} \mathrm{Al}_{2} \mathrm{O}_{3(\alpha)} \rightarrow \mathrm{TiAl}_{2} \mathrm{O}_{5(\mathrm{~s})}+\frac{4}{3} \mathrm{Al}_{(\mathrm{I})},(\Delta G=180.73-0.05 T(\mathrm{~kJ} / \mathrm{mol})) \\
& 2 \mathrm{Al}_{(\mathrm{l})}+\mathrm{TiO}_{2(\mathrm{~s})}+\frac{3}{2} \mathrm{O}_{2(\mathrm{~g})} \rightarrow \mathrm{TiAl}_{2} \mathrm{O}_{5(\mathrm{~s})}, \quad(\Delta G=-1700.1+0.32 T(\mathrm{~kJ} / \mathrm{mol}) \\
& \mathrm{Ti}_{(\mathrm{s})}+22 \mathrm{Al}_{(\mathrm{l})}+\frac{5}{2} \mathrm{O}_{(\mathrm{g})} \rightarrow \mathrm{TiAl}_{2} \mathrm{O}_{5(\mathrm{~s})}, \quad(\Delta G=-2640.1+0.5 T(\mathrm{~kJ} / \mathrm{mol}))
\end{aligned}
$$

Reaction 20 is a general form of the spinel forming equation in the phase diagram of Figure 1(d). Moreover, its Gibbs free energy of formation of the spinel is less than zero $(-18.2 \mathrm{~kJ} / \mathrm{mol}$ at $1000 \mathrm{~K}$ ), so the reaction is possible to form the spinel. However, the Gibbs free energy of reaction 21 is too high (130.73 $\mathrm{kJ} / \mathrm{mol}$ at $1000 \mathrm{~K})$. Hence, this equation cannot describe the formation of the spinel. Despite the fact that the Gibbs free energy of reaction 22 is negative and low $(-1380.1 \mathrm{~kJ} / \mathrm{mol}$ at
$1000 \mathrm{~K}$ ), it is an impossible equation, since the partial pressure of oxygen at which alumina is formed (10-47.4 atm at $1000 \mathrm{~K}$ ) and the Gibbs free energy $(-1380.1 \mathrm{~kJ} / \mathrm{mol}$ at $1000 \mathrm{~K})$ of the formation of alumina are lower than those in $\mathrm{TiO}_{2}(10-39.8 \mathrm{~atm}$ at $1000 \mathrm{~K})$. Therefore, at the $\mathrm{Al} / \mathrm{TiO}_{2}$ interface, $\mathrm{Al}_{2} \mathrm{O}_{3}$ may form when $\mathrm{TiO}_{2}$ decomposes at high temperature. In other words, if Al oxidizes to $\mathrm{Al}_{2} \mathrm{O}_{3}$ first, the reaction becomes that described by reaction 23, in which the Gibbs free energy $(2140.1 \mathrm{~kJ} / \mathrm{mol}$ at $1000 \mathrm{~K}$ ) is lower than that of reaction 22 . Therefore, reaction 23 is more possible than reaction 22 . With reference to reaction 20 , the oxygen partial pressures of the decomposition of $\mathrm{Al}_{2} \mathrm{O}_{3}$ and $\mathrm{TiO}_{2}$ at $1000 \mathrm{~K}$ are 10-47.4 and 10-39.8 atm, respectively, according to Table 2. If the partial pressure of oxygen in the system is between 10-47.4 and 10-39.8 atm, Al oxidizes to alumina, and $\mathrm{Ti}$ does not oxidize to $\mathrm{TiO}_{2}$. Therefore, reaction 23 is possible.

\section{Ti-Al Intermetallic Compounds}

In the general form of the spinel forming equation in the phase diagram of Figure $1(d)$, the reaction 21 has $\log K=0.57$ $+364.6 / T$, and the activity of $\mathrm{TiO}_{2}$ is expressed with $\log \mathrm{a}_{\mathrm{TiO} 2}$ $=-\log \mathrm{K}$ when the activity of $\mathrm{Al}_{2} \mathrm{O}_{3}$ is unity. The activity of $\mathrm{TiO}_{2}$ controls the stability of $\mathrm{TiAl}_{2} \mathrm{O}_{5}$. For example, Figure 7 shows that when activity of $\mathrm{TiO}_{2}$ is less than 0.121 at $1000 \mathrm{~K}, \mathrm{TiAl}_{2} \mathrm{O}_{5}$ will be decomposed. The partial pressure of $\mathrm{O}_{2}$ also controls the stability of $\mathrm{TiAl}_{2} \mathrm{O}_{5}$, the reaction being as 22 . For example, Figure 8 shows that when the activity of $\mathrm{Al}$ is unity, $\log \mathrm{a}_{\mathrm{TiO} 2}=$ $-0.57-364.6 / T$, and $\log P_{\mathrm{O} 2}$ is less than -33.7 atm at 1000 $\mathrm{K}, \mathrm{TiAl}_{2} \mathrm{O}_{5}$ will be decomposed. In Figures 7 and 8, when $\mathrm{a}_{\mathrm{TiO} 2}<$ 0.121 and $-33.7>\log P_{\mathrm{O}_{2}}>39.8$ atm at $1000 \mathrm{~K}$, both $\mathrm{TiAl}_{2} \mathrm{O}_{5}$ and $\mathrm{TiO}_{2}$ are stable. In contrast, when $a_{\mathrm{TiO}_{2}}<0.121$ and log $P_{\mathrm{O}_{2}}<39.8 \mathrm{~atm}$ at $1000 \mathrm{~K}, \mathrm{TiAl}_{2} \mathrm{O}_{5}$ is decomposed; when and at $1000 \mathrm{~K}$, both $\mathrm{TiAl}_{2} \mathrm{O}_{5}$ and $\mathrm{TiO}_{2}$ are decomposed. Various elements and compounds presented in the Ti-Al interfaces depend on $\mathrm{a}_{\mathrm{TiO} 2}$ and $P_{\mathrm{O} 2}$ conditions at various temperatures, such that high temperature, low oxygen pressure, and low $\mathrm{TiO}_{2}$ activity are the possible conditions for obtaining $\mathrm{Ti}_{3} \mathrm{Al}_{(s)^{\prime}}, \mathrm{TiAl}_{2(s)^{\prime}} \mathrm{TiAl}_{3(s)^{\prime}}$ $\mathrm{Ti}_{2} \mathrm{Al}_{5(\mathrm{~s})}$, and $\mathrm{TiAl}$ phases. 


\section{Conclusions}

Thermodynamic calculations were performed to estimate $\mathrm{Ti} /$ Al interface reactions under various partial oxygen pressures and the activities of titanium conditions in the Ti-Al-O system. Solid, liquid, and gas phases in the Ti-Al-O system were analyzed using thermochemical data and phase diagrams. Seven condensed phases $\left(\mathrm{Al}_{(1)^{\prime}}, \mathrm{Al}_{2} \mathrm{O}_{3(s)^{\prime}} \mathrm{TiAl}_{3(\mathrm{~s})^{\prime}} \mathrm{TiAl}_{2(\mathrm{~s})^{\prime}}, \mathrm{TiAl}_{(\mathrm{s})^{\prime}} \mathrm{Ti}_{3} \mathrm{Al}_{(\mathrm{s})^{\prime}}\right.$ and $\left.\mathrm{TiAl}_{2} \mathrm{O}_{5(\mathrm{~s})}\right)$ were arranged along an axis of a 3-domain diagram. The oxides forming in the $\mathrm{Ti} / \mathrm{Al}$ interface were dependent on the temperature, activity of $\mathrm{TiO}_{2}$, and partial oxygen pressure. When $a_{\mathrm{TiO}_{2}}>0.121$ and $\log P_{\mathrm{O} 2}>-33.7 \mathrm{~atm}$ at $1000 \mathrm{~K}$, both $\mathrm{TiAl}_{2} \mathrm{O}_{5}$ and $\mathrm{TiO}_{2}$ are stable. On the contrary, when and at $1000 \mathrm{~K}$, both $\mathrm{TiAl}_{2} \mathrm{O}_{5}$ and $\mathrm{TiO}_{2}$ were decomposed in the $\mathrm{Ti} / \mathrm{Al}$ interfaces. A 3-D diagram of Ti-Al-O isothermal stability was constructed over the temperature range of 1000 to $1200 \mathrm{~K}$. This diagram is useful for determining accurate areas of $\mathrm{TiAl}_{2} \mathrm{O}_{5}, \mathrm{Al}$, $\mathrm{Al}_{2} \mathrm{O}_{3}$ and $\mathrm{Ti} / \mathrm{Al}$ intermetallic compounds in the Ti-Al-O system. In $\mathrm{Ti} / \mathrm{Al}$ systems, $\mathrm{TiAl}$ and $\mathrm{Ti}_{3} \mathrm{Al}$ have favorable properties at elevated temperatures. According to thermodynamic calculations, low oxygen pressure, high temperature, and low $\mathrm{TiO}_{2}$ activity promote the formation of $\mathrm{TiAl}$ and $\mathrm{Ti}_{3} \mathrm{Al}$ phases.

\section{Acknowledgements}

This work was financially supported by the Chung-Shan Institute of Science and Technology (CSIST) under the Contract No. CSIST-442-V103, and NSC 102-2221-E-239-008.

\section{References}

Barin I (1989) Thermochemical Data of Pure Substances. VCH verrlagsgesellschaft mbh, New York, USA, pp.17, 18, 45-48, 1520, $1521,1544-1550$.

Braun J, and M Ellner (2001) Phase equilibria investigations on the aluminum-rich part of the binary system Ti-Al. Metall Mater Trans A 32 (5): 1037-1047.

Cai ZH, DL Zhang (2006) Sintering behaviour and microstructures of $\mathrm{Ti}(\mathrm{Al}, \mathrm{O}) / \mathrm{Al}_{2} \mathrm{O}_{3}, \mathrm{Ti}_{3} \mathrm{Al}(\mathrm{O}) / \mathrm{Al}_{2} \mathrm{O}_{3}$ and $\mathrm{TiAl}(\mathrm{O}) / \mathrm{Al}_{2} \mathrm{O}_{3}$ in situ composites. Mater Sci Eng A 419 (1-2): 310-317.

Caron M, G Gagnon, V Fortin, JF. Currie, L Ouellet,Y Tremblay, M Biberger, and R Reynolds (1996) Calculation of a Al-Ti-O-N quaternary isotherm diagram for the prediction of stable phases in TiN/AI alloy contact metallization. J Appl Phys 79 (8): 4468-4473.

Charlu TV, OJ Kleppa, and TB Reed (1974) High-temperature combustion calorimetry III. Enthalpies of formation of titanium oxides. J Chem Thermodynamics 6 (11): 1065-1074.

Chase MW Jr., CA Davies, JR Downey, Jr. DJ Furip, RA McDonald, and AN Syverud (1985) JANAF Thermochemical Tables Third Edition. American Chemical Society, New York, USA, pp. 1679-1684.

Chen YL, M Yan, YM Sun, BC Mei, and JQ Zhu (2009) The phase transformation and microstructure of $\mathrm{TiAl} / \mathrm{Ti}_{2} \mathrm{AlC}$ composites caused by hot pressing. Ceram Inter 35 (5): 1807-1812.

Chen Y, DJ Young, and B Gleeson (1995) A new Ti-rich ternary phase in the Ti-Al-O system. Mater Lett 22 (3-4): 125-129.
Cheng L, H Chang, B Tang, H Kou, and J Li (2013) Characteristics of metadynamic recrystallization of a high $\mathrm{Nb}$ containing TiAl alloy. Mater Lett 92 (1): 430-432.

Copland E (2006) Vapor pressures in the $\left\{\mathrm{Al}_{(1)}+\mathrm{Al}_{2} \mathrm{O}_{3(s)}\right\}$ system: Reconsidering $\mathrm{Al}_{2} \mathrm{O}_{3(\mathrm{~s})}$ condensation. J Chem Thermodynamics 38 (4): 443-449.

Dehm G, C Scheu, M Rühle, and R Raj (1998) Growth and structure of internal $\mathrm{Cu} / \mathrm{Al}_{2} \mathrm{O}_{3}$ and $\mathrm{Cu} / \mathrm{Ti} / \mathrm{Al}_{2} \mathrm{O}_{3}$ interfaces. Acta Mater 436 (3): 759-772.

Feng ZS, JJ Chen, C Zhang, N Zhao, and Zi Liang (2012) Formation of $\mathrm{Al}_{2} \mathrm{O}_{3}-\mathrm{TiO}_{2}$ composite oxide films on aluminum foil by cathodic electrodeposition and anodizing. Ceram Inter 38 (3): 2501-2505.

Gerasimov KB, and SV Pavlov (1996) Metastable Ti-Al phases obtained by mechanical alloying. J Alloys Compd 242 (1-2): 136-142.

Kainuma R, M Palm, and G. Inden (1994) Solid-phase equilibria in the Ti-rich part of the Ti-Al system. Intermetallics 2 (4): 321-332.

Kostov A, and D Živković (2008) Thermodynamic analysis of alloys TiAl, Ti-V, Al-V and Ti-Al-V. J Alloys Compd 460 (1-2): 164-171.

Kuo CG, WD Jehng, SJ Hsieh, and CC Chen (2009) Phase equilibria on $\mathrm{Ni}-\mathrm{Al}$ interface under low oxygen pressure. J Alloys Compd 480 (2): 299-305.

Li XL, R Hillel, F Teyssandier, SK Choi, and FJJ. Van Loo (1992) Reactions and phase relations in the Ti-Al -O system. Acta Metall Mater 40 (11): 3149-3157.

Lin CK, SS Hong, and PY Lee (2000) Formation of NiAl- $\mathrm{Al}_{2} \mathrm{O}_{3}$ intermetallic-matrix composite powders by mechanical alloying technique. Intermetallics 8 (9-1 1): 1043-1048.

Lu H, CL Bao, DH Shen, XJ Zhang, YD Cui, and ZD Lin (1995a) Study of the $\mathrm{Ti} / \mathrm{Al}_{2} \mathrm{O}_{3}$ interface. J Mater Sci 30 (2): 339-346.

Lu YC, SL Sass, Q Bai, DL Kohlstedt, and WW Gerberich (1995b) The influence of interfacial reactions on the fracture toughness of $\mathrm{Ti} /$ $\mathrm{Al}_{2} \mathrm{O}_{3}$ interfaces. Acta Metall Mater 43 (1): $31-41$.

Pang JC, GH Fan, XP Cui, AB Li, L Geng, ZZ Zheng, and QW Wang (2013) Mechanical properties of $\mathrm{Ti}-\left(\mathrm{SiC}_{\mathrm{p}} / \mathrm{Al}\right)$ laminated composite with nano-sized $\mathrm{TiAl}_{3}$ interfacial layer synthesized by roll bonding. Mater Sci Eng A 582: 294-298.

Pourbaix M (1974) Atlas of Electrochemical Equilibria in Aqueous Solutions. National Association of Corrosion Engineers, Houston, USA, pp. 213-222.

Seifert HJ, A Kussmaul, and F Aldinger (2001) Phase equilibria and diffusion paths in the Ti-Al-O-N system. J Alloys Compd 317-318 (12): 19-25.

Wang L, JX Shang, FH Wang, Y Chen, and Y Zhang (2013) Oxygen adsorption on $\gamma$-TiAl surfaces and the related surface phase diagrams: A density-functional theory study. Acta Mater 61 (5): 1726-1738.

Ward-Close CM, R Minor, and PJ Doorbar (1995) Intermetallic-matrix composite - a review. Intermetallics 4 (3): 217-229.

Wriedt HA (1985) The Al-O (Aluminum-Oxygen) system. Bull. Alloy Phase Diagrams 6 (6): 548-553.

Xu L, YY Cui, YL Hao, and R. Yang (2006) Growth of intermetallic layer in multi-laminated Ti/Al diffusion couples. Mater Sci Eng A 435-436 (5): 638-647.

Zalar A, BMM Baretzky, S Hofmann, M Rühle, and P Panjan (1999) Interfacial reactions in $\mathrm{Al}_{2} \mathrm{O}_{3} / \mathrm{Ti}_{2} \mathrm{Al}_{2} \mathrm{O}_{3} / \mathrm{Ti}_{3} \mathrm{Al}$ and $\mathrm{Al}_{2} \mathrm{O}_{3} / \mathrm{TiAl}$ bilayers. Thin Solid Films 352 (1-2): 151-155.

Zhong H, Y Yang, J Li, J Wang, T Zhang, S Li, and J Zhang (2012) Influence of oxygen on microstructure and phase transformation in high Nb containing TiAl alloys. Mater Lett 83 (1): 198-201. 